\title{
Determinants of inappropriate admissions of children to county hospitals: a cross- sectional study from rural China
}

Shi-han Lei ${ }^{1,2+}$, Yan Zhang ${ }^{1,2+}$, Hao-miao Li ${ }^{1,2}$, Dai Su ${ }^{1,2}$, Jing-jing Chang ${ }^{1,2}$, Xiao-mei Hu ${ }^{1,2}$, Qing Ye ${ }^{1,2}$, Di Jiang ${ }^{1,2}$ and Ying-chun Chen ${ }^{1,2^{*}}$ (D)

\begin{abstract}
Background: The incidence of inappropriate admissions in China has become the shackle of its' service supply system. This research aims to assess the level of children's inappropriate admissions to county hospitals in rural China and identify the characteristics and determinants of children's inappropriate admissions.

Methods: A retrospective review was conducted on data of children aged 0-14 years. A total of 771 children medical records in four county hospitals was collected by stratified random sampling in Midwestern China and was evaluated through the Rural Appropriateness Evaluation Protocol. A questionnaire survey was conducted among doctors whose names were shown in medical records. Chi-square test was used to analyse the characteristics of inappropriate admissions, and a binary logistic regression model was used to examine the determinants of inappropriate admissions.

Results: Inappropriate admissions indicate that patients who could have been treated as outpatients received services as inpatients. The average rate for inappropriate admissions of children in county hospitals was $61.35 \%$. The highest rate of inappropriate admissions was found among children aged 1-5 years (68.42\%). Inappropriate admissions mostly occurred in children with respiratory diseases (72.45\%), circulatory diseases (72.22\%) and certain infectious diseases and parasitic diseases (70.37\%). Binary logistic regression analysis showed that county, normal health status, treating department, disease, the length of hospital stay and the doctor's self-evaluation on the understanding about the degree of the patient's feelings were determinants for children's inappropriate admissions.

Conclusions: County hospitals have a high rate of inappropriate admissions of children. The relationship of children's inappropriate admissions to age distribution and the insurance compensation is affected by disease and hospitalisation expenses, respectively. The determinants of children's inappropriate admissions are directly related to the weak level of primary care services in the health service system, the initial requirements requested by children's admission decision makers and the interests among medical institutions and doctors.
\end{abstract}

Keywords: Inappropriate admission, Children, County hospital, Rural China

\footnotetext{
*Correspondence: chenyingchunhust@163.com

Shi-han Lei and Yan Zhang contributed equally to the development of this research study.

'School of Medicine and Health Management, Tongji Medical College, Huazhong University of Science and Technology, Wuhan 430030, Hubei, China

${ }^{2}$ Research center for Rural Health Services, Hubei Province Key Research Institute of Humanities and Social Sciences, Wuhan 430030, Hubei, China
}

(c) The Author(s). 2019 Open Access This article is distributed under the terms of the Creative Commons Attribution 4.0 International License (http://creativecommons.org/licenses/by/4.0/), which permits unrestricted use, distribution, and reproduction in any medium, provided you give appropriate credit to the original author(s) and the source, provide a link to the Creative Commons license, and indicate if changes were made. The Creative Commons Public Domain Dedication waiver (http://creativecommons.org/publicdomain/zero/1.0/) applies to the data made available in this article, unless otherwise stated. 


\section{Background}

Inappropriate admissions indicate that patients who could have been treated as outpatients received services as inpatients $[1,2]$. This phenomenon increases the average medical expenditure and waste in health resources [3]. Some studies have reported that inappropriate admissions have substantially contributed to high hospitalisation rate [4]. From 2003 to 2013, the hospitalisation rate for children aged 0-14 years in rural China increased by 2.86 times from 1.73 to $4.95 \%$ [5]. The increasing hospitalisation rate for children expanded faster than the forecast, suggesting that inappropriate admissions might occur. Several studies have indicated that children are the high-risk group for inappropriate admissions [6, 7]. In 2013, the rate of inappropriate admissions for children (aged 0-14 years) in county hospitals was $21.8 \%$, which was higher than that for people aged 15-64years (5.8\%) and aged 65 years and older (5.6\%) [7]. The number of children in China has increased with the universal two-child policy. Inappropriate admissions can cause cross-infection and other threats to children's health [8]. Therefore, controlling the inappropriate admissions of children is important.

As a special group in society, children lack the ability to decide whether or not they should be hospitalised. Their admission often stems from their guardians or doctors [8]. National conditions, including China's long-term one-child policy, ensure that the inappropriate admissions of children in China are different from those in other countries in some way. On the one hand, urban areas include municipalities and prefecture-level cities; rural areas include counties or county-level cities and their subordinate towns and villages. Therefore, the establishment of medical institutions in China covers municipal hospitals, county hospitals, township hospitals and village clinics. The latter three of the above-mentioned medical institutions are rural medical institutions [9]. Among them, county hospitals have the strongest capacity in providing medical services, so they play a leading role within counties. In terms of hospitalisation services, county hospitals treat more kinds of diseases than township hospitals. Meanwhile, village clinics generally do not provide inpatient services. Taking a prefecture-level city in China as an example, data show that among the pediatric inpatients classified by municipal hospitals, county hospitals, township hospitals and private hospitals, the number of hospitalised children in county hospitals accounts for $50.2 \%$ of the total number of hospitalised patients; children hospitalized in municipal hospitals and township hospitals was 39.4 and $7.3 \%$ of the total number of patients, respectively [10]. The tendency of children patients to hospitalise in county hospitals affects the rate of inappropriate admissions of children in county hospitals. On the other hand, from the perspective of service providers, doctors tend to provide extended services to be on the safe side because children cannot truly describe their feelings. This phenomenon could lead defensive medicine which influences the occurrence of inappropriate admissions $[11,12]$.

In recent years, the Chinese government has issued a series of policies, such as the payment reform of new rural cooperative medical system (NRCMS) and the family doctor contracting services, to guide patients to seek appropriate medical advices and prevent improper hospitalisation behaviours. However, the unreasonable demands and utilisations of inpatient services remain unresolved. Causes of this problem are not yet clear. It is necessary to identify the determinants of children's inappropriate admissions, so as to improve the efficiency of the utilisation of hospital services, avoid unnecessary waste of medical resources, and ensure the quality and safety of children's medical services.

Most studies on inappropriate admissions of children were based on their medical records provided by hospitals $[6,13-23]$. They mainly focused on evaluating the rate of inappropriate admissions, identifying the basic characteristics and influencing factors and further analysing health policies, hospital management system and health service behaviors behind them. In terms of characteristics, studies focused on the severity of illness and diseases in hospitalised children. For instance, De Marco et al. suggested that children with influenza-like illness are likely to have inappropriate admissions [6]. On the analysis of influencing factors, Aida et al. found that the ratio of inappropriate admissions could be reduced by strengthening the relationship between hospitals and community service agencies [15]. Magdy et al. believed that the rate of inappropriate admissions of children in Kuwait was up to $40 \%$, which can be explained by its' free health care services and easy access to hospital services [13]. In China, a few studies were conducted about inappropriate admissions on the whole population including children were conducted. Meanwhile, the existing studies did not focus on the children in county hospitals. Given differences in the determinants of inappropriate admissions of children and other groups, factors influencing inappropriate admissions of children at the county hospitals are worthy of further investigation. Therefore, this study investigated the determinants of children's inappropriate admissions. The research hypothesis of this study is that the appropriateness of children hospitalisation to county hospitals in rural China is related to the health policies and environments factors regarding children.

This study aims to investigate the level and determinants of the inappropriate admissions of children to provide comprehensive and targeted strategies for the managers and decision-makers in controlling this phenomenon.

\section{Methods}

\section{Sample selection}

We conducted this research in 2017, and the surveyed population was inpatient aged 0-14 years. In the eastern 
part of China, many rural areas rapidly became cities due to social development. Hence, county property attributes decreased. Therefore, this study did not include the eastern part of China in the research scope. We applied stratified random sampling and the principle of convenience in selected counties and county-level cities in China's central (e.g. Dingyuan in Anhui Province) and western (e.g. Weiyuan and Huining in Gansu Province; Yilong in Sichuan Province) regions, which were designated as sample areas. The biggest county hospital in each county was used as the sample hospital. Table 1 shows the basic information and the service provision of the sample county hospitals in the year before this study was carried out.

We believed that the NRCMS in different regions affected the inappropriate admissions for residents. Thus we also collected the compensation policies for NRCMS in the selected regions. Table 2 shows how the patients from the four counties benefited from the reimbursement policies of NRCMS. These counties shared the same financing policies in 2017. Reimbursement took two forms, namely, outpatient and inpatient reimbursements. The outpatient service in county hospitals was not covered. The reimbursement of inpatients ranged from 75 to $85 \%$ of the total reimbursements, and the deductible line was between 400 and 650 RMB. Overall, the NRCMS hospitalisation benefits were high in all regions.

\section{Sample size estimation}

According to previous studies, the estimated inappropriate admission rate $P$ is $40 \%$, the expected error $\delta$ is 0.035 and the confidence level $\alpha$ is 0.05 in the sampling calculation:

$$
\mathrm{N}=\mathrm{Z}_{\alpha / 2}^{2} \times P(1-P) / \delta^{2}=1.96^{2} \times 0.40 \times(1-0.40) / 0.035^{2}=753
$$

Based on the population in the surveyed areas and the scale of operation of the surveyed hospitals, 220 hospitalised children medical records were selected in Dingyuan county of Anhui Province and Yilong of Sichuan Province and 175 hospitalised children medical records were selected in Weiyuan and Huining County of Gansu Province. Children were admitted in departments such as paediatrics, surgery, orthopaedics and internal medicine. Our study selected number of medical records in each department relative to the proportion of children in the department. After the non-evaluable medical records were removed, 771 medical records of inpatients in four counties were finally obtained.

\section{Records appropriateness evaluation}

Considering the actual situation of China, 771 admission records were collected at the beginning and identified through the improving rural appropriateness evaluation protocol (RAEP) which was culturally suitable and widely used in this country (Additional file 1). There were three specially trained experts evaluated the medical records collected, all of whom were Ph.D. and involved in health policy research more than five years. Experts who owned the rich of research experience and deep understanding of existing services in rural China enabled to make the fair judgment on records with strict accordance with standards. Furthermore, each record was examined by two judges to ensure the quality of the medical records. If two judges had different opinions, the medical record would be sent to a third party. We obtained the results after a comprehensive evaluation. The RAEP standard was based on the value of the medical records. Therefore, all RAEP related indexes were extracted firstly. The actual values were then compared with the standard values. If an index value corresponded to the standard value, the admission record was appropriate. If all the relevant values of the medical record did not conform to the evaluation table criteria, the record was an inappropriate admission.

\section{Research content}

First, information, including personal and hospitalisation information of patients, was collected from the inpatient medical records. The patient's personal information

Table 1 The fundamental state of the sample county hospitals

\begin{tabular}{lllll}
\hline Index & $\begin{array}{l}\text { County hospital in } \\
\text { Dingyuan }\end{array}$ & $\begin{array}{l}\text { County hospital in } \\
\text { Weiyuan }\end{array}$ & $\begin{array}{l}\text { County hospital in } \\
\text { Huining }\end{array}$ & $\begin{array}{l}\text { County hospital in } \\
\text { Yilong }\end{array}$ \\
\hline Designated bed capacity & 845 & 350 & 400 & 750 \\
Number of actually available bed & 880 & 450 & 652 & 780 \\
$\begin{array}{l}\text { Number of on-post staff } \\
\text { Among them: }\end{array}$ & 1233 & 328 & 951 & 851 \\
$\quad$ & 319 & 81 & 157 & 198 \\
$\quad$ Doctors & 499 & 49 & 483 & 376 \\
Registered nurses & 531.5 thousand person & 101.4 thousand person & 228.4 thousand person & 278.3 thousand person \\
Frequency of outpatient and emergency visits & & & 28 thousand person & 29.4 thousand person \\
\hline
\end{tabular}




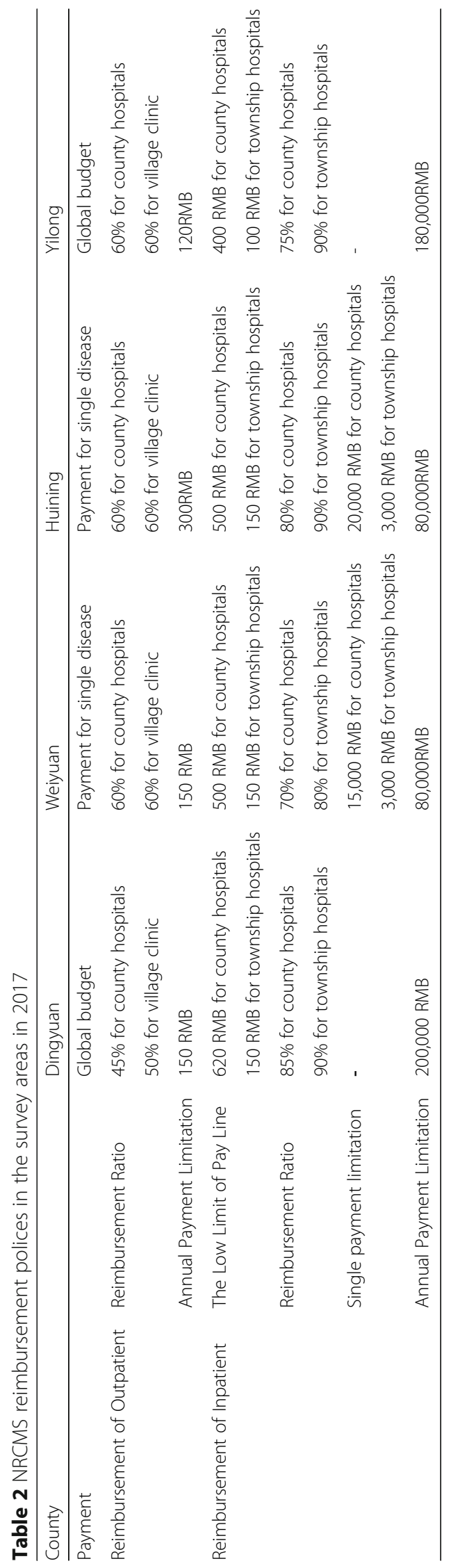


included the patient number, gender, age, normal health status and whether to get the insurance compensation. Other inpatient information included the admission department, name of the doctor in charge, day of the week of admission, emergency admission, admission and discharge diagnosis, test results, operation conditions and the length of hospital stay (LOS). After obtaining the hospitals' consent, we conducted a questionnaire survey of the doctors based on the doctor's name displayed in the inpatient medical records. The doctors' information involved age, educational background, their sense of the degree to which doctors believe they have autonomy in clinical decision-making, the degree to which doctors believe that they can understand the patient's feelings, the degree to which they think the tension in doctor-patient relationship (Additional file 2).

\section{Statistical analysis}

Medical record information of inpatients was recorded through Epidata 3.2. Diseases were classified using the international classification of diseases 10th Edition (ICD-10).

The characteristics of patients inappropriately admitted to county hospitals were analysed using Pearson's chi-square test in IBM SPSS Statistics 22.0. Given that Weiyuan County and Huining County were affiliated to the same province and considering the possible clustering between the regions, this study used the generalised estimating equation to build the model and set the job-related matrix type to unstructured firstly. However, in the correlation matrix, the main diagonal element was close to 1 , and the remaining elements were near 0 . Hence, no clustering occurred between the sampling regions. A binary logistic regression model was used to analyse the determinants of inappropriate admissions of children. The patient's admission appropriateness (appropriate $=0$, inappropriate $=1$ ) was used as the dependent variable, whereas county, sex, age, compensation for medical insurance, normal health status, admission department, day of the week of admission, disease, the admission route (admitted emergency), LOS and information of doctors mentioned in the research content were the independent variables. Backward stepwise was used to establish the regression model as follows:

$$
\begin{aligned}
\operatorname{Logit}(P)= & \ln (P / 1-P)=\beta_{0}+\beta_{1} \text { County } \\
& +\beta_{2} \text { Normal health status }+\beta_{3} \text { Department } \\
& +\beta_{4} \text { Disease }+\beta_{5} L O S \\
& +\beta_{6} \text { Doctor's understanding of patient's feelings }+\varepsilon .
\end{aligned}
$$

$P$ represents the probability of inappropriate admission for children, $\beta_{0}$ is the constant term for the model and $\varepsilon$ represents an uncontrollable random error term.

\section{Results}

Basic composition of Children's inpatients and their doctors

The first column in Table 3 shows the children's admission data: $61.09 \%$ were male, the average age was 4.25 years old (age range: $0-14$ years old), 91.83\% were patients who had gotten compensation after purchasing the NRCMS, and $89.11 \%$ were in good normal health. Most of the admissions were non-emergency, and the children with respiratory diseases accounted for $54.6 \%$ of the total number of children. The range of LOS was 133 days with average and median of 4.97 and 4.45 days, respectively.

The first column in Table 4 shows the doctor status corresponding to each medical record: 42.41 and $79.12 \%$ of records indicate that the pediatricians were greater than or equal to 46 years old and they had bachelor's degree or above. In most cases, doctors believed that they could sense patients' feelings and that they had a moderate degree of autonomy in clinical decision-making. $47.21 \%$ of the records show that doctors thought the existing doctor-patient relationship was moderate.

\section{Characteristics of children inappropriately admitted to county hospitals}

Table 3 also shows the characteristics of the patients. A total of $61.35 \%$ of the records from the four sample areas indicated inappropriate admissions. The incidence rate varied in different regions $(P<0.001)$ and ranged from 40 to $80 \%$. The Yilong County of Sichuan province had the highest rate of inappropriate admissions.

In the distribution of individual characteristics, 1 - to 5-year-old preschool children and 6- to 9-year-old primary school children were likely to be inappropriately admitted $(P<0.001)$. The children with good health and in normal status were likely to have inappropriate admissions $(P<0.001)$. No statistical difference was found in gender and inappropriate admissions, medical insurance compensation and inappropriate admissions. In terms of the distribution of inpatient characteristics, the children hospitalised in paediatrics were more likely to experience inappropriate admission than those hospitalised in other departments $(P<0.001)$. Inappropriate admission was concentrated in the children with respiratory, circulatory, infectious and digestive diseases $(P<0.001)$. The children admitted in emergency $(P=0.001)$ and those with short LOS $(P<0.001)$ were likely to have inappropriate admission. No statistical difference in the day of the week of admission was found between the two groups.

Table 4 shows that patients treated by doctors over 46-year-old were more likely to be hospitalised inappropriately than those treated by doctors under 45-year-old $(P=0.045)$. Patients treated by doctors who thought the doctor-patient relationship moderate were more likely to 
Table 3 The Appropriation Distribution Characteristics about Children Inpatients in County Hospitals According to the Medical Records

\begin{tabular}{|c|c|c|c|c|c|}
\hline \multirow[t]{2}{*}{ Variable } & \multirow[t]{2}{*}{$\mathrm{N}$ (column\%) } & \multirow{2}{*}{$\begin{array}{l}\text { Appropriate } \\
\text { N (column\%) }\end{array}$} & \multirow{2}{*}{$\begin{array}{l}\text { Inappropriate } \\
\text { N (column\%) }\end{array}$} & \multirow[t]{2}{*}{$x^{2}$} & \multirow[t]{2}{*}{$P$} \\
\hline & & & & & \\
\hline$\overline{\text { All }}$ & $771(100.00)$ & $298(38.65)$ & $473(61.35)$ & & \\
\hline \multicolumn{6}{|l|}{ County } \\
\hline Dingyuan & $219(28.40)$ & $63(28.77)$ & 156(71.23) & \multirow[t]{4}{*}{68.083} & \multirow[t]{4}{*}{$<0.001$} \\
\hline Weiyuan & 166(21.53) & $90(54.22)$ & $76(45.78)$ & & \\
\hline Huining & $171(22.18)$ & $95(55.56)$ & $76(44.44)$ & & \\
\hline Yilong & $215(27.89)$ & $50(23.26)$ & 165(76.74) & & \\
\hline \multicolumn{6}{|l|}{ Gender of patients } \\
\hline Male & $471(61.09)$ & 181(38.43) & $290(61.57)$ & \multirow[t]{2}{*}{0.025} & \multirow[t]{2}{*}{0.874} \\
\hline Female & $300(38.91)$ & 117(39.00) & 183(61.00) & & \\
\hline \multicolumn{6}{|l|}{ Age of patients, (years) } \\
\hline Less than 1 & $93(12.06)$ & $52(55.91)$ & $41(44.09)$ & \multirow[t]{5}{*}{34.947} & \multirow[t]{5}{*}{$<0.001$} \\
\hline $1-5$ & $437(56.68)$ & 138(31.58) & $299(68.42)$ & & \\
\hline $6-9$ & 151(19.58) & $56(37.09)$ & $95(62.91)$ & & \\
\hline $10-14$ & $90(11.67)$ & $52(57.78)$ & $38(42.22)$ & & \\
\hline Mean (SD) & $4.25(3.70)$ & & & & \\
\hline \multicolumn{6}{|c|}{ Whether patients get medical insurance compensation } \\
\hline Yes & 708(91.83) & 276(38.98) & $432(61.02)$ & \multirow[t]{2}{*}{0.403} & \multirow[t]{2}{*}{0.526} \\
\hline No & 63(8.17) & 22(34.92) & $41(65.08)$ & & \\
\hline \multicolumn{6}{|l|}{ Normal heath of patients } \\
\hline Good & $687(89.11)$ & 242(35.23) & $445(64.77)$ & \multirow[t]{2}{*}{31.204} & \multirow[t]{2}{*}{$<0.001$} \\
\hline General & $84(10.89)$ & $56(66.67)$ & 28(33.33) & & \\
\hline \multicolumn{6}{|l|}{ Department } \\
\hline Paediatrics & $654(84.82)$ & 212(32.42) & $442(67.58)$ & \multirow[t]{2}{*}{70.661} & \multirow[t]{2}{*}{$<0.001$} \\
\hline Others & $117(15.18)$ & $86(73.50)$ & $31(26.50)$ & & \\
\hline \multicolumn{6}{|c|}{ Day of the week of admission } \\
\hline Saturday- Sunday & 196(25.42) & $73(37.24)$ & 123(62.76) & 0.219 & 0.64 \\
\hline Monday-Friday & $575(74.58)$ & $225(39.13)$ & $350(60.87)$ & & \\
\hline Disease category & & & & & \\
\hline Respiratory disease & $421(54.6)$ & $116(27.55)$ & $305(72.45)$ & 95.079 & $<0.001$ \\
\hline Digestive disease & $94(12.19)$ & 37(39.36) & $57(60.64)$ & & \\
\hline Injury and poisoning & $42(5.45)$ & $30(71.43)$ & $12(28.57)$ & & \\
\hline Conditions originating & $56(7.26)$ & $43(76.79)$ & $13(23.21)$ & & \\
\hline in perinatal period & & & & & \\
\hline Infectious Diseases & $54(7.00)$ & 16(29.63) & $38(70.37)$ & & \\
\hline Symptoms and signs & $21(2.72)$ & $10(47.62)$ & $11(52.38)$ & & \\
\hline Circulatory disease & $18(2.33)$ & $5(27.78)$ & 13(72.22) & & \\
\hline Others & $65(8.43)$ & $41(63.08)$ & 24(36.92) & & \\
\hline Admitted emergency & & & & & \\
\hline Yes & $611(79.25)$ & 255(41.73) & $356(58.27)$ & 11.808 & 0.001 \\
\hline No & $160(20.75)$ & $43(26.88)$ & 117(73.13) & & \\
\hline
\end{tabular}


Table 3 The Appropriation Distribution Characteristics about Children Inpatients in County Hospitals According to the Medical Records (Continued)

\begin{tabular}{|c|c|c|c|c|c|}
\hline \multirow[t]{2}{*}{ Variable } & \multirow[t]{2}{*}{ N (column\%) } & \multirow{2}{*}{$\begin{array}{l}\text { Appropriate } \\
\text { N (column\%) }\end{array}$} & Inappropriate & \multirow[t]{2}{*}{$x^{2}$} & \multirow[t]{2}{*}{$P$} \\
\hline & & & N (column\%) & & \\
\hline \multicolumn{6}{|l|}{ LOS, (days) } \\
\hline Less than 5 & $400(51.88)$ & 119(29.75) & $281(70.25)$ & \multirow[t]{5}{*}{40.007} & \multirow[t]{5}{*}{$<0.001$} \\
\hline $5-8$ & 293(38.00) & 128(43.69) & $165(56.31)$ & & \\
\hline More than 9 & $78(10.12)$ & $51(65.38)$ & $27(34.62)$ & & \\
\hline Mean (SD) & $4.97(3.43)$ & & & & \\
\hline Median & 4.45 & & & & \\
\hline
\end{tabular}

be inappropriately admitted $(P=0.002)$. There is no significant difference among groups on the degree of doctor's educational backgrounds, the doctors' self-determination in diagnosis and their understanding of patients' feelings to children's inappropriate admissions.

\section{Probabilistic model of patients in children inappropriately admitted to county hospitals}

The binary logistic regression model was used to analyse the determinants of inappropriate admissions of children in county hospitals. With the minimum value as the reference level, the first and ninth variable selection results are shown in Table 5.
Based on the logistic regression analysis, the major determinants of inappropriate admissions of children to county hospitals included county, normal health status, treating department, disease LOS and the doctor's self-evaluation on the understanding about the degree of the patient's feelings. With Dingyuan County as the control group, the possibility of inappropriate admission in Weiyuan $(\mathrm{OR}=0.597)$ and Huining $(\mathrm{OR}=0.468)$ was significantly low. The children with poorer normal health were more likely to have appropriate admission $(\mathrm{OR}=0.545)$ than the children with good health. The children hospitalised in other departments had lower inappropriate hospitalisation rate than those in paediatric department $(\mathrm{OR}=0.173)$. Compared with the children with respiratory diseases, children with certain conditions

Table 4 The Appropriation Distribution Characteristics about Children Inpatients in County Hospitals under the Perception of Doctors

\begin{tabular}{|c|c|c|c|c|c|}
\hline \multirow[t]{2}{*}{ Variable } & \multirow[t]{2}{*}{ N (column\%) } & \multirow{2}{*}{$\begin{array}{l}\text { Appropriate } \\
\text { N (column\%) }\end{array}$} & Inappropriate & \multirow[t]{2}{*}{$x^{2}$} & \multirow[t]{2}{*}{$P$} \\
\hline & & & N (column\%) & & \\
\hline \multicolumn{6}{|l|}{ Age of doctors, (years) } \\
\hline Less than 36 & $207(26.85)$ & $92(44.44)$ & 115(55.56) & \multirow[t]{3}{*}{6.190} & \multirow[t]{3}{*}{0.045} \\
\hline $36-45$ & 237(30.74) & $95(40.08)$ & 142(59.92) & & \\
\hline 46 years old and above & $327(42.41)$ & 111(33.94) & $216(66.06)$ & & \\
\hline \multicolumn{6}{|c|}{ Educational background of doctors } \\
\hline Junior college and below & $161(20.88)$ & $60(37.27)$ & $101(62.73)$ & \multirow[t]{2}{*}{0.164} & \multirow[t]{2}{*}{0.685} \\
\hline Bachelor degree or above & $610(79.12)$ & 238(39.02) & $372(60.98)$ & & \\
\hline \multicolumn{6}{|c|}{ The degree to which doctors believe they have autonomy in clinical decision-making } \\
\hline Low & 115(14.92) & $48(41.74)$ & $67(58.26)$ & \multirow[t]{3}{*}{1.118} & \multirow[t]{3}{*}{0.572} \\
\hline Moderate & $355(46.04)$ & 140(39.44) & 215(60.56) & & \\
\hline High & $301(39.04)$ & 110(36.54) & 191(63.46) & & \\
\hline \multicolumn{6}{|c|}{ The degree to which doctors believe they can understand the patient's feelings } \\
\hline Low & 159(20.62) & $56(35.22)$ & 103(64.78) & \multirow[t]{3}{*}{1.085} & \multirow[t]{3}{*}{0.581} \\
\hline Moderate & $577(72.24)$ & 229(39.69) & $348(60.31)$ & & \\
\hline High & $35(4.54)$ & 13(37.14) & $22(62.86)$ & & \\
\hline \multicolumn{6}{|c|}{ The degree to which doctors believe tensions between doctors and patients } \\
\hline Low & 184(23.87) & $91(49.46)$ & $93(50.54)$ & \multirow[t]{3}{*}{12.777} & \multirow[t]{3}{*}{0.002} \\
\hline Moderate & $364(47.21)$ & 123(33.79) & $241(66.21)$ & & \\
\hline High & 223(28.92) & $84(37.67)$ & 139(62.33) & & \\
\hline
\end{tabular}


Table $\mathbf{5}$ Logistic regression analysis of inappropriate admission of children inpatients $(n=771)$

\begin{tabular}{|c|c|c|c|c|c|}
\hline Variable & $\mathrm{AOR}$ & $95 \% \mathrm{Cl}$ & Variable & AOR & $95 \% \mathrm{Cl}$ \\
\hline \multicolumn{3}{|l|}{$\overline{\text { Step-1 }}$} & \multirow{2}{*}{\multicolumn{3}{|c|}{$\begin{array}{l}\text { The degree to which doctors think } \\
\text { tensions between doctors and patients (Low) }\end{array}$}} \\
\hline \multicolumn{3}{|l|}{ County (Dingyuan) } & & & \\
\hline Weiyuan & 0.685 & $0.355-1.321$ & \multirow[t]{3}{*}{ Moderate } & 0.823 & $0.433-1.567$ \\
\hline Huining & 0.506 & $0.275-0.929$ & & 0.654 & \multirow[t]{2}{*}{$0.297-1.440$} \\
\hline Yilong & 2.116 & $1.056-4.239$ & & 5.930 & \\
\hline Gender of patients (Male) & 0.976 & $0.686-1.389$ & \multicolumn{3}{|l|}{ Step-9 } \\
\hline \multicolumn{3}{|l|}{ Age of patients, (years) (Less than 1) } & \multicolumn{3}{|l|}{ County (Dingyuan) } \\
\hline $1-5$ & 1.688 & $0.920-3.098$ & Weiyuan & 0.597 & $0.346-1.031$ \\
\hline $6-9$ & 1.472 & $0.724-2.995$ & Huining & 0.468 & $0.281-0.777$ \\
\hline $10-14$ & 1.204 & $0.539-2.690$ & Yilong & 1.752 & $1.034-2.969$ \\
\hline \multirow{2}{*}{$\begin{array}{l}\text { Whether patients get insurance } \\
\text { compensation (Yes) }\end{array}$} & \multirow[t]{2}{*}{1.275} & \multirow{2}{*}{$0.663-2.452$} & Normal heath (Good) & 0.545 & $0.305-0.974$ \\
\hline & & & Department (Paediatrics) & 0.173 & $0.092-0.324$ \\
\hline Normal heath of patients (Good) & 0.562 & $0.309-1.024$ & Day of the week of admission & 0.690 & $0.465-1.024$ \\
\hline Department (Paediatrics) & 0.185 & $0.094-0.363$ & (Satu & & \\
\hline \multirow{2}{*}{$\begin{array}{l}\text { Day of the week of admission } \\
\text { (Saturday- Sunday) }\end{array}$} & \multirow[t]{2}{*}{0.685} & \multirow[t]{2}{*}{$0.459-1.023$} & \multicolumn{3}{|l|}{ Disease category (Respiratory disease) } \\
\hline & & & Digestive disease & 1.305 & $0.728-2.339$ \\
\hline \multicolumn{2}{|l|}{ Disease category (Respiratory disease) } & & Injury and poisoning & 0.401 & $0.165-0.970$ \\
\hline Digestive disease & 1.248 & $0.686-2.272$ & Conditions originating in perinatal period & 0.215 & $0.104-0.446$ \\
\hline Injury and poisoning & 0.346 & $0.142-0.844$ & Infectious Diseases & 1.058 & $0.539-2.077$ \\
\hline Conditions originating in perinatal period & 0.245 & $0.115-0.523$ & Symptoms and signs & 0.536 & $0.206-1.396$ \\
\hline Infectious Diseases & 1.053 & $0.533-2.082$ & Circulatory disease & 1.219 & $0.335-4.438$ \\
\hline Symptoms and signs & 0.458 & $0.172-1.218$ & Others & 0.553 & $0.279-1.098$ \\
\hline Circulatory disease & 1.304 & $0.341-4.979$ & LOS, (days) (Less than 5) & & \\
\hline Others & 0.556 & $0.276-1.120$ & $5-8$ & 0.642 & $0.440-0.936$ \\
\hline Admitted Emergency (Yes) & 1.095 & $0.654-1.834$ & More than 9 days & 0.501 & $0.266-0.945$ \\
\hline \multicolumn{3}{|l|}{ LOS, (days) (Less than 5) } & \multirow{2}{*}{$\begin{array}{l}\text { The degree to which doctors believe } \\
\text { they can understand the patient's } \\
\text { feelings (Low) }\end{array}$} & & \\
\hline $5-8$ & 0.634 & $0.432-0.932$ & & & \\
\hline More than 9 & 0.505 & $0.266-0.958$ & Moderate & 0.697 & $0.446-1.091$ \\
\hline \multicolumn{2}{|l|}{ Age of doctors, (years) (Less than 36) } & & High & 2.302 & $0.862-6.149$ \\
\hline $36-45$ & 1.646 & $0.929-2.915$ & Constant & 6.694 & \\
\hline
\end{tabular}

Educational background of doctors (Junior college and below)

The degree to which doctors believe they have autonomy in clinical decision-making (Low)

\section{Moderate}

$0.307-1.301$

$$
\text { High }
$$

The degree to which doctors believe they can understand the patient's feelings (Low)

Moderate

Table 5 Logistic regression analysis of inappropriate admission of children inpatients $(n=771)$ (Continued)

originating from perinatal period had a lower likelihood of inappropriate admissions $(\mathrm{OR}=0.215)$. Moreover, the children with LOS of $5-8$ days $(\mathrm{OR}=0.642)$ and 9 days or more $(\mathrm{OR}=0.501)$ or whose doctor believed that he/she could understand the patient's feelings moderately $(\mathrm{OR}=$ 0.697) had the lower risk of inappropriate admissions than those others.

\section{Discussion}

Inappropriate admissions of children in county hospitals in rural China

In 2017, the rate of inappropriate admissions of children aged $0-14$ years in Chinese county hospitals was $61.35 \%$, which was higher than the rate in other countries such as $40.7 \%$ in Kuwait (2012), 29\% in Canada (1993) and 
$23 \%$ in Sweden (2004) [13, 20, 21]. Based on the previous statistics, the differences in inappropriate admission rates were due to the differences in the specific age groups of children in each study and the differences in health service systems among these countries. Firstly, China has not yet adopted a mandatory system of primary care, so patients have high autonomy in the choice of institutions for medical treatment [23]. Secondly, China is implementing family doctor contracting services, which usually means that a service team composed of township hospitals and village clinic doctors should provide basic public health services and medical guidance to rural residents to improve their health behaviors and to clarify medical care [24]. However, most of the primary medical institutions in China focus on the provision of basic public health services projects rather than guiding patients to seek medical service reasonably. This easily leads patients to select a relatively high-quality county hospital for treatment, rather than choosing the primary medical institution that also be capable to diagnose and treat the disease.

The $61.35 \%$ rate of children's inappropriate admission in this study is higher than that in previous (2013). This change is due to the difference between the policy environments of each study time and the children's characteristics. For one thing, changes in policy environments may lead to an increase in the rate of inappropriate admissions through changes in the interest-driven mechanism of medical institutions. For another, children have vulnerabilities, and their diseases differ from those of other populations [25]. Risk aversion refers to the strategy of taking the initiative to abandon or change measures to avoid the risks when the possibility of risk loss is high. Therefore, when children's diseases symptoms are mainly characterized by long-term cold, fever, diarrhoea and abdominal pain, the parents tend to have their children hospitalised. Moreover, because relations between doctors and patients are more strained than before. Doctors will consider that parents are more sensitive to the treatment mood of children, that is, out of concern for children, parents are more anxious when their child is sick, and that the instability of outpatient infusion treatment, i.e. the side effects of infusion treatment are great. They may admit children who do not need hospitalisation. This phenomenon also increases the possibility of inappropriate admissions.

\section{Characteristics of children who were hospitalised inappropriately}

The characteristics including gender, disease, treating department, admission in the emergency, the day of the week of admission and LOS in the hospital were consistent with those in the previous research results [13-23]. Except for gender, the day of the week of admission, educational background of doctors, doctors' view on their decision-making power and their understanding of patients' feelings, the other variables had remarkable differences in the appropriateness of hospitalisation of the children. In the present study, the rate of inappropriate admission of 0- to 1-year old (infant stage) is low, which occurs because infants are likely to be hospitalised for preterm birth, congenital problems and infectious diseases [16]. Zhang Yan et al. pointed out that the NRCMS reimbursement design directly determines the choice of medical service and leads to the patient's moral hazard. In this study, however, there is no correlation between obtaining medical insurance compensation and rationality of children's admission [22, 26]. Previous studies also showed that when inpatient and outpatient costs were similar, the patients decided to hospitalise [27]. Therefore, the low cost of hospitalisation for children, in certain degree, can explain the relationship between medical compensation and children's inappropriate admissions.

\section{Determinants of Children's patients who were hospitalised inappropriately}

County, normal health status, department, disease, LOS and the degree to which doctors believe they can understand the patient's feelings significantly affect the appropriateness of the children's hospitalisation in county hospitals in rural China (Table 5). International experience shows that the mode of medical insurance payment determines the mode of operation of medical providers, thereby affecting the inappropriate admissions [28-30]. Therefore, the differences of NRCMS payment methods in the investigated areas led to the discrepancies among county hospitals. In this study, the payment method in Dingyuan and Yilong is the global budget, which emphasises reducing the inappropriate services through total amount control. This may cause the county hospital to inhale a large number of patients who should be hospitalised in the township hospital since the county hospital is the centre of the rural medical system. The payment method in Weiyuan and Huining is payment for the single disease, which emphasises the reduction of inappropriate inducement by controlling single diseases. However, there may be doctors who decompose hospitalised patients with comorbidities, resulting in inappropriate admissions. Therefore, the medical insurance department should increase supervision and scientifically promote the reform of medical insurance fund payment.

Because of lacking the mastery of medical expertise, children with poorer normal health status are less prone to inappropriate admissions. On the one hand, when children who rarely get sick suddenly become ill, their parents will have excessive care, which may result in excessive demand for children's inpatient services [8]. On the other hand, some children who are not in good normal health had experienced medical treatments. Parents of these children have an increased understanding of 
reasonable treatment methods, thus reducing the generation of inappropriate services. As a result, we can reduce the occurrence of inappropriate admissions by strengthening the management of children's health and guiding parents to seek correct medical service. Consistent with most studies, the treating department has an effect on inappropriate admissions [31]. Paediatric departments are likely to have inappropriate admissions due to the internal management of hospitals. The management problems are reflected in the admission criteria and the incentive mechanism. For one thing, most of the children hospitalised in other departments are surgical patients; hence, their needs for hospitalisation are easy to generate. For another, given that the assessment of doctors is linked to the number of admissions, the doctor's personal salary is linked to the business volume, and the admission criteria are not unified. Under the background of information asymmetry between doctors and patients, doctors are prone to induce demand and thus affect hospitalisation [32-34]. Therefore, we should pay attention to the management of paediatric departments and define the admission criteria for future management. Disease to inappropriate admissions is consistent with the previous research results. The main diseases of children are common diseases, such as bronchopneumonia and upper respiratory tract infection [6]. Most of these diseases have not reached the standards of admission; hence, the rate of inappropriate admission is high. Meanwhile, the outpatient department has different treatments and control levels for different system diseases. For example, cases originated from the perinatal period and child nursing-sensitive situations are not well controlled in the outpatient department. Hospitalisation is more appropriate than outpatient care under these circumstances [35]. The inpatient's LOS affects the occurrence of inappropriate admissions. Accordingly, the inappropriate admission rate can be reduced by controlling the first diagnosis at township hospitals or community-based medical institutions.

\section{Limitations}

The shortcomings of this study are as follows: Firstly, this study is a cross-sectional study due to the limited investigation time. A panel data structure for the children admitted inappropriately is impossible to establish. The conclusions may not be comprehensive enough. Secondly, the international standard of Paediatrics Appropriateness Evaluation Protocol was established for the appropriateness of children's admission, but to judge whether or not these standards are applicable to county hospitals in rural China is impossible. This study takes RAEP to evaluate the hospitalisation records of children in county hospitals in China, so there may be deviations in the criteria for different populations. Thirdly, the content of the doctor's survey is not rich enough, and further research is needed in the future.

\section{Conclusions}

According to RAEP, the incidence of inappropriate admission of children to county hospitals in Chinese rural areas is high. This high incidence is directly related to the weak level of primary care services in the health service system, the initiative requirements of the children's admission decision makers, the interests of the medical institutions and doctors. This study found that the government can control inappropriate admissions of children from the following aspects: 1) Improve the rural health service system. The inappropriate admissions of children can be controlled by improving children's disease diagnosis and treatment in township hospitals and the level of primary medical treatment and two-way referrals. 2) Strengthen the leading role of medical insurance. Firstly, we should integrate the interests of medical service providers in the reformation and promotion of the mode of payment. Secondly, the role of medical insurance in the admission supervision of medical institutions should be strengthened. The regulatory content includes a clear admission standard. 3) Reinforce the health management of children. The primary medical staff can guide the parents' effective medical treatment by educating the children's guardians on the prevention and treatment of children's common diseases to reduce the inappropriate admission rate.

\section{Additional files}

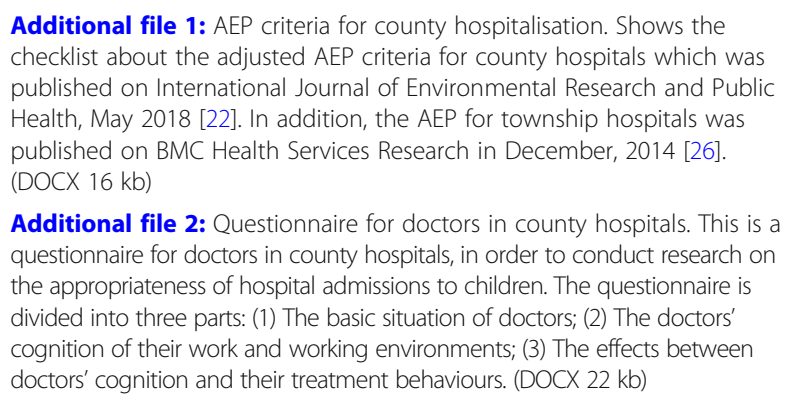

Additional file 1: AEP criteria for county hospitalisation. Shows the checklist about the adjusted AEP criteria for county hospitals which was published on International Journal of Environmental Research and Public Health, May 2018 [22]. In addition, the AEP for township hospitals was published on BMC Health Services Research in December, 2014 [26]. (DOCX 16 kb)

Additional file 2: Questionnaire for doctors in county hospitals. This is a questionnaire for doctors in county hospitals, in order to conduct research on the appropriateness of hospital admissions to children. The questionnaire is divided into three parts: (1) The basic situation of doctors; (2) The doctors' cognition of their work and working environments; (3) The effects between doctors' cognition and their treatment behaviours. (DOCX $22 \mathrm{~kb}$ )

Abbreviations

LOS: Length of hospital stay; NRCMS: New rural cooperative medical system; RAEP: Rural appropriateness evaluation protocol

\section{Acknowledgements \\ The authors thank the Health and Family Planning Commission of Weiyuan county, Gansu province, for their willingness to provide us the data.}

\section{Funding}

This work was supported by the National Natural Science Foundation of China (NO. 71473096). The funders had no role in study design, data collection and analysis, decision to publish or preparation of the manuscript.

Availability of data and materials

The datasets used and/or analysed during the current study are available from the corresponding author on reasonable request. Contact information: chenyingchun@hust.edu.cn. 


\section{Authors' contributions}

S-H.L. and Y.Z. and Y-C.C. contributed to the conception and design of the project; S-H.L. and H-M.L and J-J.C. contributed to the analysis and interpretation of the data; D.S. and X-M.H. and Q.Y. contributed to the data acquisition and provided statistical analysis support; S-H.L. and Y.Z. drafted the article. S-H.L. and Y.Z. and D.J. revise the manuscript All authors supplied critical revisions to the manuscript and gave final approval of the version to be published.

\section{Ethics approval and consent to participate}

The research methods and investigation tools in this study were approved by the Ethics Committee of Tongji Medical College, Huazhong University of Science and Technology (IORG No: IORG0003571). The patient information was anonymized and deidentified before the analysis. The informed consent was obtained from all participants in written.

\section{Consent for publication}

Not applicable.

\section{Competing interests}

The authors declare there are no competing interests.

\section{Publisher's Note}

Springer Nature remains neutral with regard to jurisdictional claims in published maps and institutional affiliations.

\section{Received: 2 September 2018 Accepted: 1 February 2019} Published online: 18 February 2019

\section{References}

1. Lavis JN, Anderson GM. Appropriateness in health care delivery: definitions, measurement and policy implications. CMAJ. 1996;154:321-8.

2. Payne SM. Identifying and managing inappropriate hospital utilization: a policy synthesis. Health Serv Res. 1987;22(5):709.

3. Fusco M, Buja A, Piergentili P, et al. Individual and hospital-related determinants of potentially inappropriate admissions emerging from administrative records. Health Policy. 2016;120(11):1304-12. https://doi.org/ 10.1016/j.healthpol.2016.09.015.

4. Restuccia J, Shwartz M, Ash A, et al. High hospital admission rates and inappropriate care. Health Aff. 1996;15(4):156-63. https://doi.org/10.1377/ hlthaff.15.4.156.

5. National Health and Family Planning Commission Statistical Information Centre. 2013 fifth National Health Service Survey and analysis reports [M]//. China: Peking Union Medical College Press; 2015.

6. De Marco G, Mangani S, Correra A, Di Caro S, Tarallo L, De Franciscis A, Jefferson T, Guarino A. Reduction of inappropriate hospital admissions of children with influenza-like illness through the implementation of specific guidelines: a case-controlled study. Pediatrics. 2005;116:e506-11. https://doi. org/10.1542/peds.2005-0053.

7. Zhu S. The study of characteristics and influencing factors of inappropriate hospital admissions in the new rural cooperative medical system. Huazhong Univ Sci Technol. 2013. https://doi.org/10.7666/d.D415008.

8. Chen YC. Excessive demand for inpatient Services in Rural Areas: measurement and Management of Inappropriate Admissions [M]. China: China Science Publishing \& Media Ltd. (CSPM); 2014.

9. National Health and Family Planning Commission. 2017 China Health and Family Planning Statistical Yearbook [J] 2017.

10. Huang XW, Wu ZJ, Liu JS, Chen YG. Discuss the hospital grading management according to the flow orientation of hospitalization children. Mod Hosp. 2011;9:75-7. https://doi.org/10.3969/j.issn.1671-332X.2011.09.038.

11. Restuccia J, Shwartz M, Ash A, et al. High hospital admission rates and inappropriate care. Health Aff. 1996;15(4):156. https://doi.org/10.1126/ science.644329.

12. Summerton N. Positive and negative factors in defensive medicine: a questionnaire study of general practitioners. BMJ. 1995;310(6971):27-9. https://doi.org/10.1136/bmj.310.6971.27.

13. Shafik MH, Seoudi TMM, Raway TS, et al. Appropriateness of pediatric hospitalization in a general Hospital in Kuwait. Med Princ Pract. 2012;21(6): 516-21. https://doi.org/10.1159/000339084.

14. Formby DJ, Mcmullin ND, Danagher $K$, et al. The appropriateness evaluation protocol: application in an Australian children's hospital. Aust Clin Rev. 1991;11(4):123.
15. Bianco A, Pileggi C, Trani F, et al. Appropriateness of admissions and days of stay in pediatric wards of Italy. Pediatrics. 2003;112(1 Pt 1):124. https://doi.org/10.1542/peds.112.1.124.

16. Esmain A, Quayle JA, Roberts C. Assessing the appropriateness of paediatric hospital admissions in the United Kingdom. J Public Health Med. 2000;22(2): 231-8. https://doi.org/10.1093/pubmed/22.2.231.

17. Vincitorio D, Chiaradia G, Waure CD, et al. Appropriateness of admission and days of stay in pediatric hospital in Ancona, Italy. J Public Health. 2010;18(5): 497-503. https://doi.org/10.1007/s10389-010-0325-y.

18. Katz M, Warshawsky SS, Porat A, et al. Appropriateness of pediatric admissions to a tertiary care facility in Israel. Isr Med Assoc J. 2001;3(3):501-3.

19. Havens PL, Butler JC, Day SE, et al. Treating measles: the appropriateness of admission to a Wisconsin children's hospital. Am J Public Health. 1993;83(3): 379. https://doi.org/10.2105/AJPH.83.3.379.

20. Smith HE, Sheps S, Matheson DS. Assessing the utilization of in-patient facilities in a Canadian pediatric hospital. Pediatrics. 1993;92(4):587-93.

21. Thollander J, Gertow O, Hansen S, et al. [Assessment of inappropriate emergency admissions. A study of 566 consecutive cases][J]. Lakartidningen. 2004;101(10):888.

22. Zhang $Y$, Zhang $L$, Li $H$, et al. Determinants of inappropriate admissions in county hospitals in rural China: a cross-sectional study. Int J Environ Res Public Health. 2018;15(6):1050. https://doi.org/10.3390/ijerph15061050.

23. Zhang $Y$, Tang $W X$, Sun XW, Hu R, Zhang L. Studying on the influence of residents' seeing a doctor freely on the integration of health service system in China. Chinese Health Serv Manage 2014; 9: 678-680. doi: 1004-4663(2014)09-678-03

24. Pan $\mathrm{CL}$, Yang JX, CH XU. Impact of rural doctors' signing service on health literacy among rural residents in Dafeng district of Jiangsu province. Chin J Public Health. 2018;2:214-7. https://doi.org/10.11847/zgggws1114274.

25. Chen WH. Research of characteristic and medical care cost of inpatient in a children hospital: Central South University; 2016. https://doi.org/10.7666/d. y1083969.

26. Zhang $Y$, Chen $Y$, Zhang $X$, et al. Current level and determinants of inappropriate admissions to township hospitals under the new rural cooperative medical system in China: a cross-sectional study. BMC Health Serv Res. 2014;14(1):649. https://doi.org/10.1186/s12913-014-0649-3.

27. Xin YJ, Xiang L. Analysis of the inpatient service demand and medical security level of children in the rural area: a case study of Xiantao City, Hubei Province. Chinese J Health Policy. 2017;2:69-73. https://doi.org/10. 3969/j.issn.1674-2982.2017.02.012

28. Rice T. Physician payment policies: impacts and implications. Annu Rev Public Health. 1997;18(18):549. https://doi.org/10.1146/annurev.publhealth. 18.1.549.

29. Barros PP. Cream-skimming, incentives for efficiency and payment system. J Health Econ. 2003;22(3):419-43. https://doi.org/10.1016/S0167-6296(02)00119-4.

30. Dumont $\mathrm{E}$, Fortin $\mathrm{B}$, Jacquemet $\mathrm{N}$, et al. Physicians' multitasking and incentives: empirical evidence from a natural experiment. J Health Econ. 2008:27(6):1436-50. https://doi.org/10.1016/j.jhealeco.2008.07.010.

31. Ferrero BO, Sanchez ML, Corredera RC, Uriarte AE, de Miguel DLVF. Inappropriate admissions to the Department of Internal Medicine evaluated by the AEP (appropriateness evaluation protocol). An Med Interna. 1998;15:674-5.

32. Evans RG. Supplier-induced demand: some empirical evidence and implications [M]// the economics of Health and medical care. UK: Palgrave Macmillan; 1974. https://doi.org/10.1007/978-1-349-63660-0_10.

33. Gaynor M, Hass-Wilson D, Voge WB. Are Invisible Hands Good Hands? Moral Hazard, Competition, and the 2nd Best in Health Care Markets. SSRN Electronic Journal. 1998;108(5):992-1005. https://doi.org/10.2139/ssrn.135855.

34. Prezotto KH, Chaves MM, Mathias TA. Hospital admissions due to ambulatory care sensitive conditions among children by age group and health region. Revista Da Escola De Enfermagem Da Usp. 2015;49(1):44-53. https://doi.org/10.1590/S0080-623420150000100006.

35. Kemper KJ. Medically inappropriate hospital use in a pediatric population. N Engl J Med. 1988;318(16):1033-7. https://doi.org/10.1056/NEJM198804213181605. 